\title{
钛酸钡/硅酸钙复合生物活性压电陶瓷的制备及性能研究
}

\author{
魏子钦 ${ }^{1,2}$, 夏 翔 $^{2}$, 李 勤 $^{2}$, 李国荣 ${ }^{2}$, 常 江 ${ }^{1,2}$ \\ (1. 上海师范大学 化学与材料科学学院, 上海 200234; 2. 中国科学院 上海硅酸盐研究所, 高性能陶瓷与超微结 \\ 构国家重点实验室，上海 200050)
}

摘 要: 压电材料产生的电信号能够促进成骨细胞增殖分化，但不具有良好的诱导矿化能力; 生物活性材料在生理 环境下能够诱导类骨羟基磷灰石的沉积, 但又不能产生电信号促进成骨。因此, 开发出一种既能产生电信号, 又能 诱导矿化沉积的复合生物活性压电材料, 具有重要意义。本研究以铁酸钡为压电组分, 以硅酸钻为生物活性组分, 采用固相烧结法制备了钛酸钡/硅酸钙复合生物活性压电陶瓷，测试了压电性能，并用体外矿化实验评价了诱导矿 化能力。硅酸钻复合含量达到 $30 \%$ 时, 复合陶瓷仍具有一定的压电性能 $\left(d_{33}=4 \mathrm{pC} \cdot \mathrm{N}^{-1}\right)$, 并且能够在模拟体液中诱导 磷酸钻沉积。钛酸钡与硅酸钙的复合能够同时具有压电性和生物活性，为骨修复材料提供了新的选择。

关 键 词: 钛酸钡; 硅酸钻; 压电性; 生物活性

中图分类号: TQ174 文献标志码: A

\section{Preparation and Properties of Barium Titanate/Calcium Silicate Composite Bioactive Piezoelectric Ceramics}

\author{
WEI Ziqin ${ }^{1,2}$, XIA Xiang ${ }^{2}$, LI Qin ${ }^{2}$, LI Guorong ${ }^{2}$, CHANG Jiang ${ }^{1,2}$
}

(1. College of Chemistry and Materials Science, Shanghai Normal University, Shanghai 200234, China; 2. State Key Laboratory of High Performance Ceramics and Superfine Microstructure, Shanghai Institute of Ceramics, Chinese Academy of Sciences, Shanghai 200050, China)

\begin{abstract}
Electrical signals generated by piezoelectric materials can promote proliferation and differentiation of osteoblasts, but they can't induce mineralization, while bioactive materials can induce the deposition of bone like hydroxyapatite in physiological environment, but can not generate electrical signal to promote osteogenesis. Therefore, it is of great significance to develop a composite bioactive piezoelectric material that can not only generate electrical signals, but also induce mineralization and deposition. Here, we used barium titanate as piezoelectric component and calcium silicate as bioactive component to prepare barium titanate/calcium silicate composite as bioactive/piezoelectric ceramics by solid-state sintering method. Piezoelectric properties of the ceramics were tested, and the ability of inducing mineralization was evaluated by in vitro mineralization experiment. The experimental results show that when the content of calcium silicate reaches $30 \%$, the composite ceramics still have certain piezoelectric property $\left(d_{33}=4 \mathrm{pC} \cdot \mathrm{N}^{-1}\right)$, and can induce the deposition of calcium phosphate in simulated body fluid. Therefore, the combination of barium titanate and calcium silicate can synchronously afford piezoelectric and biological activities, which provides a new choice for bone repair materials.
\end{abstract}

Key words: barium titanate; calcium silicate; piezoelectricity; bioactivity

收稿日期：2021-08-28; 收到修改稿日期：2021-10-12; 网络出版日期：2021-11-12

基金项目: 国家重点研发计划(2016YFC1100201) National Key Research and Development Plan of China (2016YFC1100201)

作者简介：魏子钦(1996-), 男, 硕士研究生. E-mail: 1149057072@qq.com WEI Ziqin (1996-), male, Master candidate. E-mail: 1149057072@qq.com

通信作者：常 江, 研究员. E-mail: jchang@mail.sic.ac.cn CHANG Jiang, professor. E-mail: jchang@mail.sic.ac.cn 
骨缺损是临床上常见的创伤问题, 少量骨缺损 可以自行生长愈合, 而大段骨缺损往往需要植入人 工骨替代材料 ${ }^{[1-3]}$ 。在骨修复过程中, 细胞的增殖分 化有着至关重要的作用, 能够促进成骨细胞增殖分 化的材料对骨修复有着明显的促进作用 ${ }^{[4]}$ 。

研究发现, 天然骨骼, 由于骨胶原蛋白的中心 不对称性, 存在压电信号, 能促进成骨细胞的增殖 分化对新骨的生成有着良好的促进作用 ${ }^{[5-7]}$ 。已有研

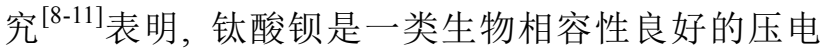
材料, 其产生的压电信号可以促进成骨细胞的增殖 分化，已被用于骨修复领域的研究。

骨骼再生是一个复杂的过程, 不仅需要有成骨 细胞的增殖分化, 还需要磷酸钙的矿化沉积, 为细 胞的迁移黏附等活动提供支持, 从而逐渐形成具有 特定结构和功能的骨骼 ${ }^{[3,12-13]}$ 。钛酸钡的矿化能力较 差，限制了其在骨修复领域的实际应用。硅酸䥻是 一种生物活性良好的生物陶瓷材料, 植入体内后能 够释放出活性的 $\mathrm{Ca}^{2+} 、 \mathrm{SiO}_{3}{ }^{2-}$ 离子, 诱导矿化, 在材 料表面沉积类骨羟基磷灰石，促进新骨生成，在骨 修复领域具有良好的应用潜力 ${ }^{[14-18]}$ 。

因此, 本研究设计将硅酸钙与钛酸钡复合, 既 可保持钛酸钡压电性能又同时赋予材料诱导矿化的 生物活性，从而提升其骨修复能力。目前尚未见钛 酸钡等压电材料兼具直接促进矿化作用的研究报 道。基于此, 本研究采用传统的固相烧结技术, 制备 了钛酸钡/硅酸钙复合生物活性压电陶瓷, 并探究了 其压电性和体外诱导矿化的能力。

\section{1 实验方法}

\section{1 实验材料}

实验所用原料为钛酸钡粉体(BT, 粒径 $4 \mu \mathrm{m}$, 纯度为 $99.9 \%$, 上海阿拉丁生化科技股份有限公司), 硅酸钙 $(\mathrm{CS}$, 纯度 $>98 \%$, 昆山华侨科技新材料有限公 司), 聚乙烯醇(PVA, 1799 型，醇解度: 98\% 99\% (摩尔 分数), 上海阿拉丁生化科技股份有限公司)。

\section{2 复合陶瓷的制备}

本课题采用固相烧结法合成了不同摩尔比例的 $\mathrm{BT} / \mathrm{CS}$ 复合陶瓷, 分别为 $\mathrm{BT} 、 \mathrm{BT}: \mathrm{CS}=9: 1$ (以下记 为 $0.9 \mathrm{BT} 0.1 \mathrm{CS}$ ) $\mathrm{BT}: \mathrm{CS}=8: 2$ (以下记为 $0.8 \mathrm{BT} 0.2 \mathrm{CS}$ )、 $\mathrm{BT}: \mathrm{CS}=7: 3$ (以下记为 $0.7 \mathrm{BT} 0.3 \mathrm{CS}$ )、 $\mathrm{BT}: \mathrm{CS}=$ $6: 4$ (以下记为 $0.6 \mathrm{BT} 0.4 \mathrm{CS}$ )、 $\mathrm{CS}$, 图 1 为简要合成 工艺流程。首先, 按照表 1 所示配比分别称取 BT 粉末、CS 粉末于玛瑙研钵中, 加入 $4 \%$ 质量分数的 PVA 溶液，充分研磨后，过孔径 $200 \mu \mathrm{m}$ (80 目)笁;

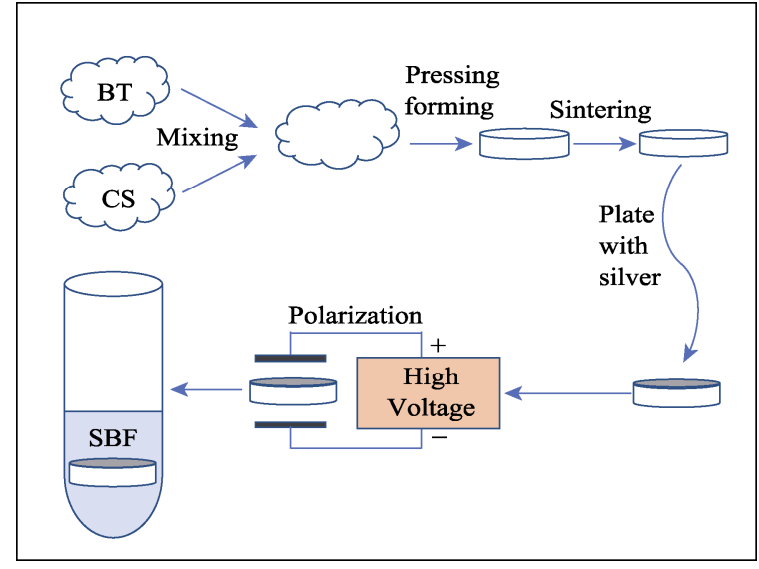

图 1 工艺流程示意图

Fig. 1 Flow chart of sample preparation

然后在 $10 \mathrm{MPa}$ 的压力下压制成直径为 $13 \mathrm{~mm}$ 、高 为 $1 \mathrm{~mm}$ 的生坏片, 之后马弗炉中进行两段升温烧 结: 以 $1{ }^{\circ} \mathrm{C} / \mathrm{min}$ 的升温速率升温至 $550{ }^{\circ} \mathrm{C}$, 保温 $4 \mathrm{~h}$, 进行排胶处理, 随后再以 $3{ }^{\circ} \mathrm{C} / \mathrm{min}$ 升温至相应 烧结温度, 保温 $4 \mathrm{~h}$, 待自然冷却至室温。根据预实 验确定不同组分的烧结温度, BT、0.9BT0.1CS、 $0.8 \mathrm{BT} 0.2 \mathrm{CS} 、 0.7 \mathrm{BT} 0.3 \mathrm{CS} 、 0.6 \mathrm{BT} 0.4 \mathrm{CS}$ 和 $\mathrm{CS}$ 的烧 结温度分别为 $1300 、 1200 、 1125 、 1110 、 1100$ 和 $1150{ }^{\circ} \mathrm{C}$ 。

\section{3 复合陶瓷的极化}

压电陶瓷烧成以后, 需要在高压直流电场下极 化处理, 以激发其压电性。将制得的复合陶瓷表面 打磨光滑, 控制厚度为 $1 \mathrm{~mm}$ 。清洗烘干后, 在其上 下表面刷一层厚薄均匀的导电银浆, 以 $2{ }^{\circ} \mathrm{C} / \mathrm{min}$ 升 温至 $720{ }^{\circ} \mathrm{C}$, 保温 $10 \mathrm{~min}$, 然后自然冷却至室温。 镀银电极后的陶瓷在硅油中以 $3 \mathrm{kV} / \mathrm{mm}$ 的电场强 度极化 $30 \mathrm{~min}$ 后取出, 使用乙醇超声清洗掉表面 的硅油。

\section{4 压电性能表征}

电滞回线和压电常数是压电陶瓷常用的表征参 数。复合陶瓷镀银后，先使用德国 aixACCT TF2000 铁电综合测试系统测试其电滞回线, 以初步判断其 压电性能, 随后在 $3 \mathrm{kV} / \mathrm{mm}$ 的高压电场下对其极化, 并采用中国科学院声学研究所生产的 ZJ-4AN 型准 静态 $d_{33}$ 测量仪测试其压电常数 $d_{33}$, 以表征材料最

表 1 复合陶瓷的原料组成

Table 1 Raw material composition of the composite ceramics

\begin{tabular}{|c|c|c|c|c|c|}
\hline BT & $\begin{array}{l}0.9 \mathrm{BT} \\
0.1 \mathrm{CS}\end{array}$ & $\begin{array}{l}0.8 \mathrm{BT} \\
0.2 \mathrm{CS}\end{array}$ & $\begin{array}{l}0.7 \mathrm{BT} \\
0.3 \mathrm{CS}\end{array}$ & $\begin{array}{l}0.6 \mathrm{BT} \\
0.4 \mathrm{CS}\end{array}$ & CS \\
\hline $\mathrm{BT} / \mathrm{g} 2.0000$ & 1.8951 & 1.7782 & 1.6481 & 1.5014 & 0 \\
\hline $\mathrm{CS} / \mathrm{g} 0$ & 0.1049 & 0.2215 & 0.3519 & 0.4986 & 2.0000 \\
\hline
\end{tabular}


终的压电性能。

\section{5 体外矿化实验}

对复合陶瓷进行体外模拟矿化实验以验证复合 陶瓷的生物活性。将极化后的陶瓷表面银电极层磨 去并清洗干净、烘干。按 Kokubo 等 ${ }^{[19]}$ 报道配置 SBF 溶液，将烘干后的复合陶瓷置于离心管中，按照公 式(1)的比例加入 $\mathrm{SBF}$ 溶液,

$$
V_{\mathrm{s}}=S_{\mathrm{a}} / 10
$$

其中, $V_{\mathrm{S}}$ 为 $\mathrm{SBF}$ 的体积 $(\mathrm{mL}), S_{\mathrm{a}}$ 为陶瓷的表面积 $\left(\mathrm{mm}^{2}\right)$ 。之后将离心管置于 $37{ }^{\circ} \mathrm{C}$ 恒温摇床中, 每 $48 \mathrm{~h}$ 更换液体, 取浸泡 $14 \mathrm{~d}$ 的样品, 经超声水洗和 醇洗，然后烘干备用。

\section{6 生物相容性表征}

本实验选用人体成纤维细胞 (Human diploid fibroblast, HDF)验证材料的细胞相容性, 具体操作 步骤如下:

1)将 BT 和 0.7BT0.3CS 陶瓷粉体用细胞培养液 配制成 $200 \mathrm{mg} \cdot \mathrm{mL}^{-1}$ 标准浸提液, 随后过滤菌膜并 梯度稀释至所需浓度。

2)用不同浓度的细胞浸提液在 $37{ }^{\circ} \mathrm{C}$ 、二氧化碳 $\left(\mathrm{CO}_{2}\right)$ 浓度 $5 \%$ 的细胞培养箱培养 $\mathrm{HDF}$ 。培养 $24 \mathrm{~h}$ 后, 用 Cell Counting Kit-8 检测 HDF 细胞活性。

细胞实验重复三次且各组数据均经过 $t$ 检验, $p<0.05$ 和 $p<0.01$ 时, 将两组数据分别看作有显著差 异和极显著差异。

\subsection{XRD、SEM、EDS 表征}

采用 $18 \mathrm{~kW}$ 转靶 X 射线衍射仪(XRD)对烧结的 陶瓷样品进行物相表征, 采用场发射扫描电子显微 镜(SEM)SU8220 观察浸泡 SBF 前后样品的微观形 貌, 并对表面进行能谱(EDS)表征, 以分析其元素 组成和比例。

\section{2 结果与讨论}

\section{1 复合陶瓷的物相分析}

复合陶瓷的 XRD 图谱如图 2(a)所示, 不同组分 的陶瓷的衍射峰主要对应于四方相 $\mathrm{BaTiO}_{3}(\mathrm{PDF} \# 79-$ 2264)和 $\mathrm{CaSiO}_{3}(\mathrm{PDF} \# 89-6463)$, 峰形的变化基本与 组分含量变化保持一致, 当 $\mathrm{BT}$ 含量较多、CS 含量 较少时, XRD 主要显示了 $\mathrm{BT}$ 的峰, 随着 $\mathrm{CS}$ 含量逐 渐提升, CS 的峰逐渐增多。未发现明显的第三相, 表明在陶瓷的烧结过程中, BT 与 CS 并未发生反应。 各组分的峰形均较为尖锐, 表明在实验选取的烧结 温度下，陶瓷的结晶度良好。

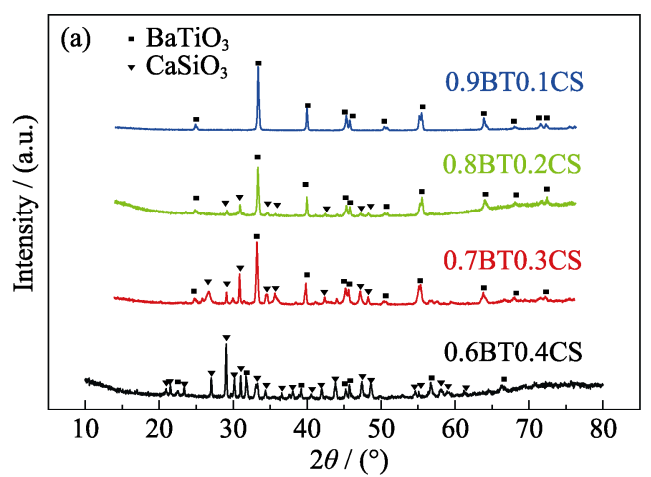

(b)
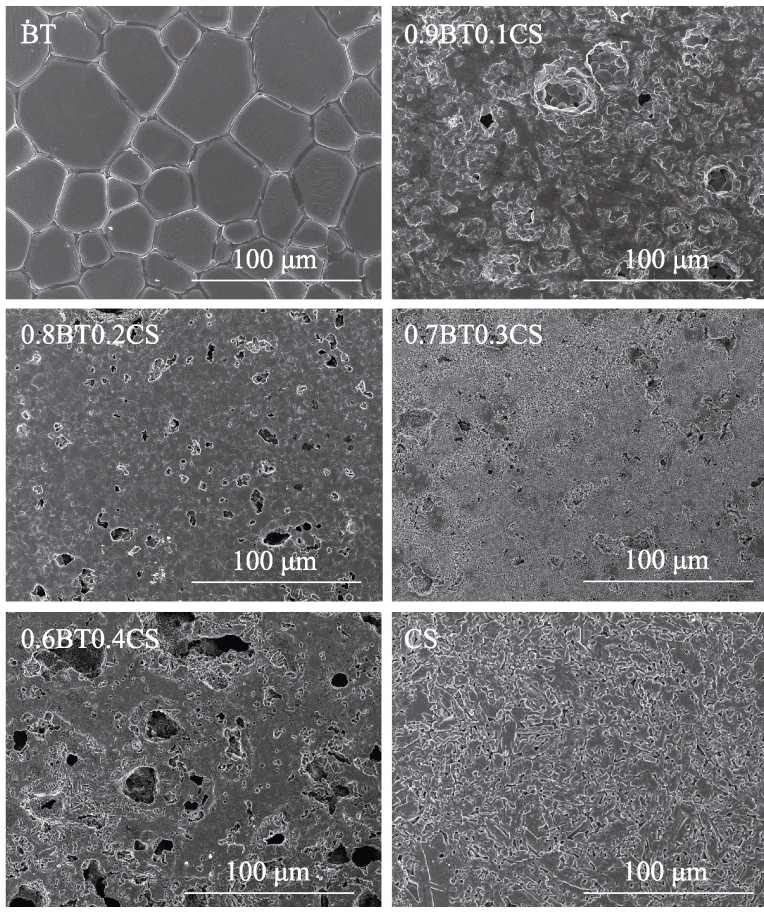

图 2 复合陶瓷的 XRD 图谱(a)及其 SEM 照片(b)

Fig. 2 XRD patterns (a) and SEM images (b) of composite ceramics

不同组分陶瓷的 SEM 照片如图 2(b)所示, BT 是 致密的陶瓷，随着 $\mathrm{CS}$ 复合含量的增加，复合陶瓷的 致密程度明显下降，这可能是由 $\mathrm{BT}$ 与 $\mathrm{CS}$ 两者烧结 收缩率的差异而导致的。此外, 由于不同组分烧结 温度不同, BT 陶瓷的烧结温度最高, 使得 $\mathrm{BT}$ 陶瓷 的晶粒明显大于其他组分。陶瓷的压电性能受烧结 温度、晶粒大小、致密度等多种因素的影响 ${ }^{[20]}$, 再 加上复合比例不同，在这几种因素的共同作用下， 不同组分的复合陶瓷的压电性能表现出较大的差异 (表 2)。

\section{2 复合陶瓷压电性能表征}

图 3 展示了复合陶瓷的压电性能表征结果。如 图 3(a)所示, 纯 BT 的 P-E 曲线显现出明显的弛豫铁 电体特征, 而图 3(f) 所示的 CS 的 P-E 曲线不具有铁 电体的特征。在 $\mathrm{BT}$ 中掺入少量 $\mathrm{CS}$ 后，复合陶瓷仍 然具有铁电特性(图 3(b d ))。不过随着 CS 含量逐渐 增大, 复合陶瓷的铁电性逐渐减弱, 当 CS 复合含 
表 2 复合陶瓷的压电常数 $\boldsymbol{d}_{33}$

Table 2 Piezoelectric constant $d_{33}$ of composite ceramics

\begin{tabular}{lcccccc}
\hline & BT & $0.9 \mathrm{BT} 0.1 \mathrm{CS}$ & $0.8 \mathrm{BT} 0.2 \mathrm{CS}$ & $0.7 \mathrm{BT} 0.3 \mathrm{CS}$ & $0.6 \mathrm{BT} 0.4 \mathrm{CS}$ & $\mathrm{CS}$ \\
\hline Before mineralization $/\left(\mathrm{pC} \cdot \mathrm{N}^{-1}\right)$ & 169 & 44 & 11 & 4 & 1 & 0 \\
After mineralization $/\left(\mathrm{pC} \cdot \mathrm{N}^{-1}\right)$ & 161 & 39 & 8 & 3 & 0 & 0 \\
\hline
\end{tabular}
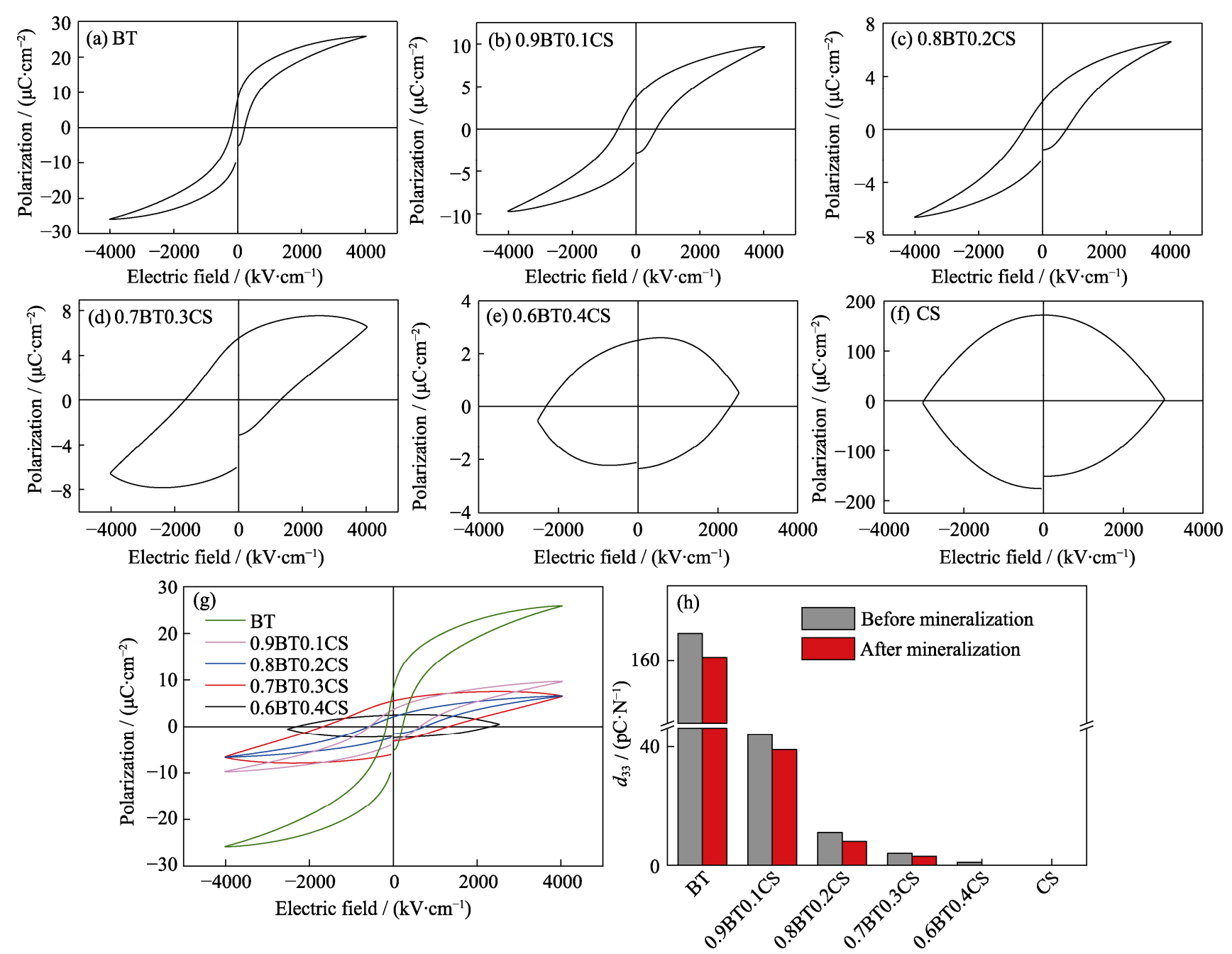

图 3 复合陶瓷的压电性能表征

Fig. 3 Characterization of piezoelectric properties of composite ceramics

(a) BT; (b) 0.9BT0.1CS; (c) 0.8BT0.2CS; (d) 0.7BT0.3CS; (e) 0.6BT0.4CS; (f) CS; (g) Variation trend of hysteresis loop; (h) Piezoelectric constant Colorful figures are available on website

量达到 40\%时几乎完全消失(图 3(e))。从图 3(g)可以 看到各组分的铁电性随 CS 掺杂含量增大而呈明显 降低的趋势。

图 3(h)和表 2 展示了复合陶瓷的压电常数 $d_{33}$ 测试结果。纯 $\mathrm{BT}$ 的 $d_{33}$ 为 $169 \mathrm{pC} \cdot \mathrm{N}^{-1}$, 显示出较强 的压电性能。CS 的 $d_{33}$ 为 0 , 表明其完全不具有压 电性。当掺入 $10 \%$ 的 CS 以后, 复合陶瓷的 $d_{33}$ 迅速 下降至 $44 \mathrm{pC} \cdot \mathrm{N}^{-1}$, 随着 $\mathrm{CS}$ 含量进一步增大, 复合 陶瓷的 $d_{33}$ 显著下降, 当 $\mathrm{CS}$ 含量达到 $40 \%$ 时, 复合 陶瓷的 $d_{33}$ 仅为 $1 \mathrm{pC} \cdot \mathrm{N}^{-1}$ 。这是因为引入 $\mathrm{CS}$ 以后，其 均匀分散在复合陶瓷中, 降低了 $\mathrm{BT}$ 相的连续性, 进 而使得在外力作用下因形变而产生的电荷聚集到陶
瓷的表面能力下降, 最终表现为压电性能下降 ${ }^{[21-22] 。}$ 此外, 实验测试了矿化后的陶瓷的压电常数 $d_{33}$, 发 现不同组分复合陶瓷的压电性能均有一定程度的下降, 但 CS 复合比例 $\leqslant 30 \%$ 的复合陶瓷的压电常数(表 2 )仍 然大于骨骼的压电常数 $\left(0.2 \mathrm{pC} \cdot \mathrm{N}^{-1}\right)^{[23]}$, 因此可以认为 矿化后的复合陶瓷仍具备足够的压电性能。

根据 Baxter 等 ${ }^{[11,24]}$ 的研究结果来看, 在与非压 电材料复合的过程中, BT 含量低于 $70 \%$ 的复合陶瓷 将不再具有压电性能, 这与本实验压电测试的实验 结果一致, 而少量复合非压电陶瓷组分仍有可能保 留陶瓷材料的压电性能, 虽然压电性会明显下降。 考虑到生物应用对于压电性能的要求明显低于常规 
应用, 这种复合仍然具有骨生理学意义 ${ }^{[25]}$ 。除了复 合陶瓷需要保留一定的压电特性之外，加入的 CS 能否赋予复合陶瓷生物活性, 是其是否具应用价值 的另一个关键。因此，为了证实制得的复合材料不 仅具有压电性，还具有诱导矿化的生物活性，本实 验对具有压电性的复合陶瓷 $0.9 \mathrm{BT} 0.1 \mathrm{CS}$ 、 $0.8 \mathrm{BT} 0.2 \mathrm{CS} 、 0.7 \mathrm{BT} 0.3 \mathrm{CS}$ 进行了矿化实验，探究其 在模拟体液中诱导沉积羟基磷灰石的能力。

\section{3 体外矿化结果分析}

图 4 为复合陶瓷在 SBF 中浸泡前后的 SEM 照 片和 EDS 扫描结果。从图 4(a h) 可以看出, 浸泡 SBF $14 \mathrm{~d}$ 以后, BT、0.9BT0.1CS 和 $0.8 \mathrm{BT} 0.2 \mathrm{CS}$ 的表 面并没有明显的矿化产物，而 $0.7 \mathrm{BT} 0.3 \mathrm{CS}$ 的表面有 一层蠕虫状沉积物。随后对矿化后的陶瓷表面进行 元素分析, 结果如图 4(i 1)与表 3 所示, BT 的表面完 全没有 $\mathrm{Ca}$ 和 $\mathrm{P}$, 而 $0.9 \mathrm{BT} 0.1 \mathrm{CS}$ 和 $0.8 \mathrm{BT} 0.2 \mathrm{CS}$ 的表 面仅有微量的 $\mathrm{P}$ 元素, 但 $\mathrm{Ca}$ 元素相对较多, 使得

$\mathrm{Ca} / \mathrm{P}$ 的比值较大。从表 3 可以看出, $0.8 \mathrm{BT} 0.2 \mathrm{CS}$ 表 面的 $\mathrm{Ca}$ 含量约为 $0.9 \mathrm{BT} 0.1 \mathrm{CS}$ 的 2 倍, 这与陶瓷本 身的 $\mathrm{Ca}$ 含量差值几乎保持一致, 这说明此处 $\mathrm{Ca}$ 的 主要来源为陶瓷本身, 并不是由矿化沉积产生。而 0.7BT0.3CS 矿化后表面的主要元素为 $\mathrm{Ca}$ 和 $\mathrm{P}$, 其 $\mathrm{Ca} / \mathrm{P}$ 为 1.27 , 这与文献[26]报道的硅酸钙陶瓷在 SBF 中浸泡诱导早期钙磷沉积的钻磷比相近。这个 结果说明，在 BT 中 CS 的掺入量达到 $30 \%$ 的情况下, 复合陶瓷不仅具有一定压电性能，而且具有一定的 诱导矿化沉积磷酸钙的生物活性。

\section{4 生物相容性}

本实验对 $\mathrm{BT}$ 和复合后矿化效果最好的 0.7BT0.3CS 进行了细胞相容性实验, 结果如图 5 所 示。与对照组相比, BT 对 HDF 的生长几乎没有影响。 而 $0.7 \mathrm{BT} 0.3 \mathrm{CS}$ 浸提液在稀释倍数为 $1 / 16$ 时对 $\mathrm{HDF}$ 的 生长有着显著的促进作用。由此可知, BT 具有良好的 细胞相容性，与 CS 复合后表现出一定的生物活性。

$14 \mathrm{~d}$
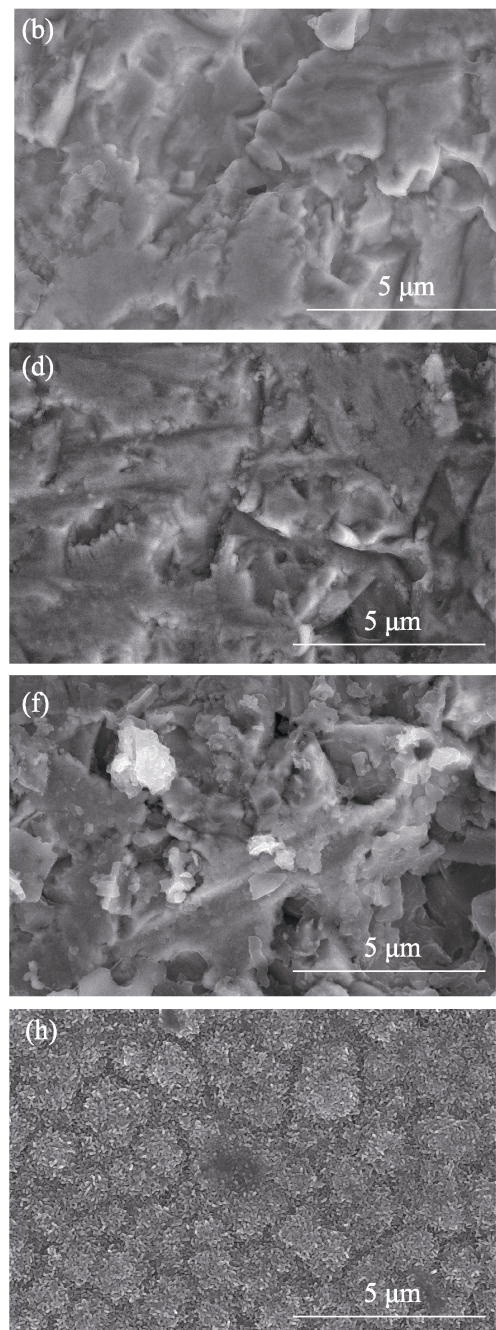

EDS
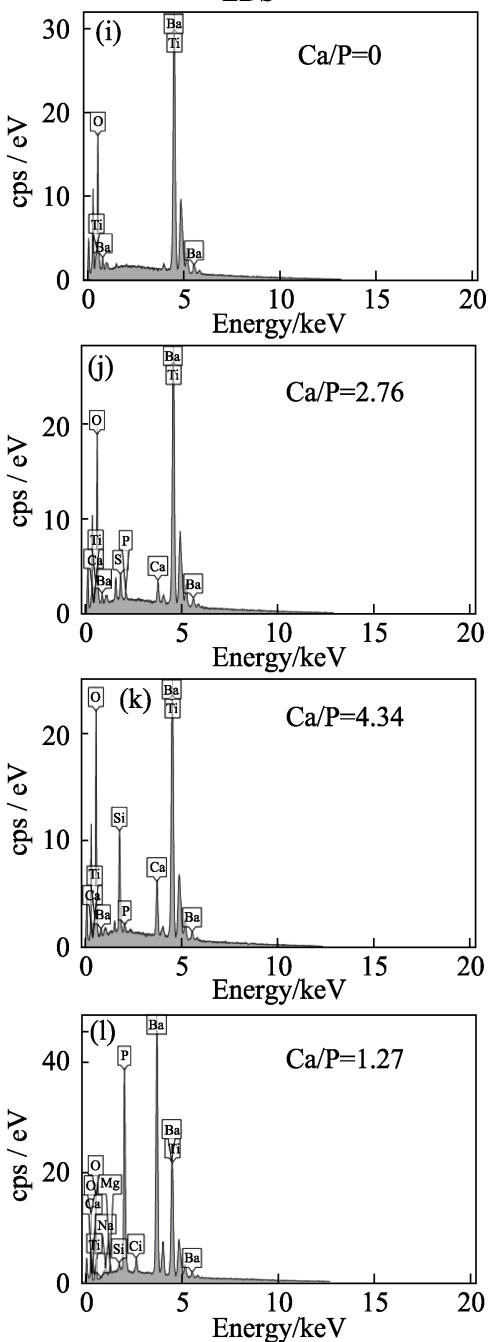

图 4 体外矿化表征

Fig. 4 Characterization of in vitro mineralization (a-h) SEM images of different ceramics soaked in SBF for 0 and $14 \mathrm{~d}$; (i-h) EDS spectra of different ceramics soaked in SBF for $14 \mathrm{~d}$ SBF: Simulated body fluid 
表 3 样品矿化 $14 \mathrm{~d}$ 后的表面元素组成

Table 3 Surface element composition of sample after $14 \mathrm{~d}$ mineralization

\begin{tabular}{ccccc}
\hline & BT $\%$ & $0.9 B T 0.1 \mathrm{CS} / \%$ & $0.8 \mathrm{BT} 0.2 \mathrm{CS} / \%$ & $0.7 \mathrm{BT} 0.3 \mathrm{CS} / \%$ \\
\hline $\mathrm{O}$ & 59.98 & 60.53 & 61.37 & 7.84 \\
$\mathrm{P}$ & - & 0.74 & 0.65 & 6.81 \\
$\mathrm{Si}$ & - & 2.57 & 7.34 & 0.23 \\
$\mathrm{Ca}$ & - & 2.04 & 4.12 & 8.62 \\
$\mathrm{Ti}$ & 19.97 & 17.38 & 14.42 & 2.65 \\
$\mathrm{Ba}$ & 20.05 & 16.74 & 12.10 & 2.71 \\
$\mathrm{Ca} / \mathrm{P}$ & - & 2.76 & 6.34 & 1.27 \\
\hline
\end{tabular}

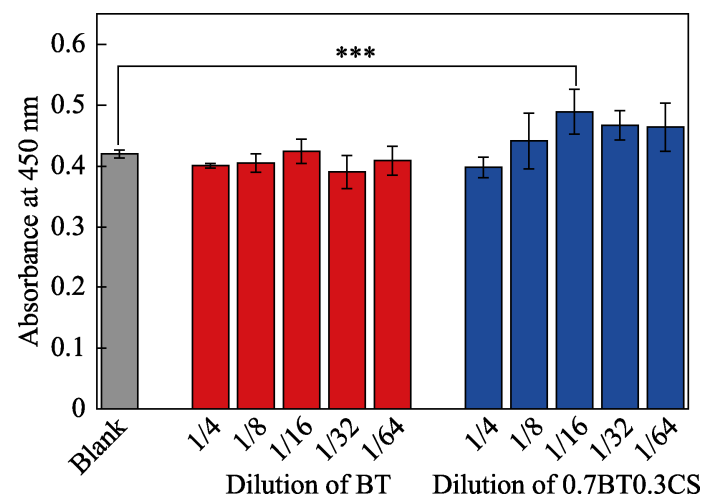

图 5 不同稀释度的 $\mathrm{BT}$ 和 $0.7 \mathrm{BT} 0.3 \mathrm{CS}$ 浸提液对 $\mathrm{HDF}$ 细胞 活力影响

Fig. 5 Cell activity of series solutions of extracts from BT and $0.7 \mathrm{BT} 0.3 \mathrm{CS}$ by CCK8 assay

$* * *$ indicates $p<0.01$

\section{3 结论}

本研究采用固相烧结法制备了不同比例的钛酸 钡/硅酸钙复合陶瓷, 通过对其压电性和体外生物活 性的研究可获以下结论:

1)在不同的复合比例下，随着硅酸钻含量增大， 复合陶瓷的压电性能逐渐下降, 但在硅酸钙复合含量 $30 \%$ 、钛酸钡含量 $70 \%$ 时仍保留一定的压电性。

2)相比于完全不具有诱导矿化沉积的钛酸钡材 料，复合 $30 \% \mathrm{CS}$ 的复合陶瓷(0.7BT0.3CS)有一定的体 外诱导矿化并且促进 HDF 细胞增殖的生物活性。

综上可知，在生物惰性的钛酸钡压电陶瓷材料 中引入生物活性的硅酸钙材料, 当复合比例处于一 定范围内，材料可以兼具压电性和诱导矿化的生物 活性。因此, 钛酸钡/硅酸钙生物压电复合材料具有 一定的骨修复应用潜力, 但其实际应用效果还需进 一步的生物学实验验证。

\section{参考文献:}

[1] ANTALYA H, JOHANNA B, RUSTOM L E, et al. Bone regeneration strategies: engineered scaffolds, bioactive molecules and stem cells current stage and future perspectives. Biomaterials,
2018, 180: 143-162.

[2] LOBB D C, DEGEORGE B R, CHHABRA A B. Bone graft substitutes: current concepts and future expectations. The Journal of Hand Surgery, 2019, 44(6): 497-505.

[3] MAAZOUZ Y, CHIZZOLA G, DOBELIN N, et al. Cell-free, quantitative mineralization measurements as a proxy to identify osteoinductive bone graft substitutes. Biomaterials, 2021, 275: 120912.

[4] HENCH L L, POLAK J M. Third-generation biomedical materials. Science, 2002, 295(5557): 1014-1017.

[5] FUKADA E, YASUDA I. On the piezoelectric effect of bone. Journal of the Physical Society of Japan, 1957, 12(10): 1158-1162.

[6] BASSETT C A L, BECKER R O. Generation of electric potentials by bone in response to mechanical stress. Science, 1962, 137(3535): 1063-1064.

[7] FUKADA E, YASUDA I. Piezoelectric effects in collagen. Japanese Journal of Applied Physics, 1964, 3(8): 117-121.

[8] PARK J B, RECUM A F V, KENNER G H, et al. Piezoelectric ceramic implants-a feasibility study. Journal of Biomedical Materials Research, 1980, 14(3): 269-277.

[9] ATTILIO M, JONATHAN B, GIUSEPPE D V, et al. Two-photon lithography of 3D nanocomposite piezoelectric scaffolds for cell stimulation. ACS Applied Materials \& Interfaces, 2015, 7(46): 25574-25579.

[10] BUSUIOC C, OLARET E, STANCU I C, et al. Electrospun fibre webs templated synthesis of mineral scaffolds based on calcium phosphates and barium titanate. Nanomaterials, 2020, 10: 772 .

[11] KHARE D, BASU B, DUBEY A K. Electrical stimulation and piezoelectric biomaterials for bone tissue engineering applications. Biomaterials, 2020, 258: 120280-1-25.

[12] WEINER S, ADDADI L. Crystallization pathways in biomineralization. Annual Review of Materials Research, 2011, 41: 21-40.

[13] REZNIKOV N, STEELE J A M, FRATZL P, et al. A materials science vision of extracellular matrix mineralization. Nature Reviews Materials, 2016, 1(8): 16041.

[14] WU C T, CHANG J. Silicate bioceramics for bone tissue regeneration. Journal of Inorganic Materials, 2013, 28(1): 29-39.

[15] LIN K L, CHANG J, WANG Z. Fabrication and the characterisation of the bioactivity and degradability of macroporous calcium silicate bioceramics in vitro. Journal of Inorganic Materials, 2005, 20(3): 692-698.

[16] LIU X Y, DING C X, WANG Z Y. Apatite formed on the surface of plasma-sprayed wollastonite coating immersed in simulated body fluid. Biomaterials, 2001, 22(14): 2007-2012.

[17] WANG X, ZHOU Y, XIA L, et al. Fabrication of nano-structured calcium silicate coatings with enhanced stability, bioactivity and osteogenic and angiogenic activity. Colloids Surf. Biointerfaces, 2015, 126: 358-366.

[18] WANG S G, XU Y C, ZHOU J, et al. In vitro degradation and surface bioactivity of iron-matrix composites containing silicatebased bioceramic. Bioactive Materials, 2017, 2(1): 10-18.

[19] KOKUBO T, TAKADAMA H. How useful is SBF in predicting in vivo bone bioactivity? Biomaterials, 2006, 27(15): 2907-2915.

[20] LI H T, ZHANG B P, WEN J B, et al. Influences of sintering temperature on structure and properties of Cu-doped lead-free LNKN ceramics. Journal of Functional Materials, 2011, 42(S5): 931-934.

[21] ZHANG S, YU F, GREEN D J. Piezoelectric materials for high temperature sensors. Journal of the American Ceramic Society, 2011, 94(10): 3153-3170.

[22] TANG Y, WU C, WU Z, et al. Fabrication and in vitro biological properties of piezoelectric bioceramics for bone regeneration. Scientific Reports, 2017, 7: 43360.

[23] KIM D, HAN S A, KIM J H, et al. Biomolecular piezoelectric materials: from amino acids to living tissues. Advanced Materials, 2020, 32(14): 1906989.

[24] BAXTER F R, TURNER I G, BOWEN C R, et al. The structure and properties of electroceramics for bone graft substitution. Key Engineering Materials, 2008, 361(22): 99-102.

[25] MAEDA H, TSUDA K, FUKADA E. Dependence on temperature and hydration of piezoelectric, dielectric and elastic-constants of bone. Japanese Journal of Applied Physics, 1976, 15(12): 2333-2336.

[26] SALAHINEJAD E, BAGHJEGHAZ M J. Structure, biomineralization and biodegradation of $\mathrm{Ca}-\mathrm{Mg}$ oxyfluorosilicates synthesized by inorganic salt coprecipitation. Ceramics International, 2017, 43(13): 10299-10306. 